\title{
Ar plasma jet generation and its application for water and surface sterilization
}

\author{
Fada Feng ${ }^{1}$, Xianjun $\mathrm{He}^{1}$, Yingying Liang ${ }^{1}$, Fengzhu Yang ${ }^{1}$, Fushan Wang ${ }^{1}$, Yue Yang ${ }^{2}$, and Tiejian Liu ${ }^{3, *}$ \\ ${ }^{1}$ Jiaying University, School of Chemistry and Environment, 514015 Meizhou City Guangdong province, China \\ ${ }^{2}$ Yangjiang Polytechnic, Department of food and Environmental Engineering, 529566 Yangjiang City Guangdong province, China \\ ${ }^{3}$ The Third Affiliated Hospital of Southern Medical University, Department of neurosurgery, 510630 Guangzhou City Guangdong \\ province, China
}

\begin{abstract}
Microorganisms are widely distributed in the living environment of human, animals and plants. Some of the microorganisms are harmful for their pathogenic effects. Non-thermal plasma technology, especially, the atmospheric pressure plasma jet, is considered to be one of the promising technologies for sterilization. This work proposes a double-dielectric barrier discharge reactor for Ar plasma jet generation. Charteristics of discharge and temperature thermogram of plasma jet are investigated by the means of U-P curve and infrared image, respectively. Performance of the plasma jet is evaluated by surface and water sterilization. The results show that, Ar plasma jet is generated stable with double-dielectric barrier discharge. The length of plasma jet increases as the applied voltage, frequency or gas flow increased, but the plasma jet generation can be restricted in high frequency or gas flow. For E. coli in the water and surface, high sterilization efficiency is observed for a short time treatment by Ar plasma jet.
\end{abstract}

\section{Introduction}

Microorganism, discovered by Leeuwenhoek in 1675, includes bacteria, fungi, archaea, and protists. They are widely distributed in human activities, animals and plants, and the natural environment. A Lot of microorganisms have pathogenicity. The pathogenic microorganisms are harmful, since they invade and grow within other organisms, causing diseases that lead to food deterioration, skin diseases, tooth decay, respiratory infections, gastrointestinal infectious diseases and so on[1].

With regard to the pathogenic microorganism sterilization, according to the type and degree of the objects to be sterilized and the environment, there are many traditional sterilization methods, such as physical sterilization, chemical sterilization, and biological sterilization[2, 3]. Physical sterilization is mainly through the extreme environment to destroy the physical structure of the bacteria for sterilization that includes heat sterilization, ultraviolet sterilization, microwave sterilization, and ionizing radiation sterilization[4]. Chemical sterilization is the method that uses various chemical disinfectants to kill the microorganisms with very high efficiency. However, the toxic disinfectant residual is still a problem for the application of chemical sterilization[5]. In the past decades, the biological sterilization is considered to be an environmentalfriendly sterilization method that has the advantages of safe, low carbon, and no pollution. But its application needs high environmental requirements.
Plasma is characterized by the existence of electrons, ions, as well as highly excited atoms, radicals, and molecules in mixture with the other neutrals of gaseous systems. In non-thermal plasma, the energy of electron can be as high as 1-20 eV[6]. Lots of active species such as positive ions, negative ions, and free radicals are generated by the effect of high energy electrons. These active species, especially, the free radicals such as reactive oxygen species (ROS) and reactive nitrogen species (RNS) can cause destruction of cell structure, cell membrane, protein, nucleic acid, and DNA, causing inactivation or death of viruses and microorganisms[7]. Actually, the ROS and RNS have been found to have an effect of inducing apoptosis of cancer cells[8].

Plasma medical research has a history of more than 50 years. The atmospheric pressure plasma jet has been widely used in caries treatment[9], skin disinfection, wound treatment and skin tumour treatment $[10,11]$. The plasma jet is considered to be a promising technology for various application, such as hydrophilicity and chemical activity modification of materials[12], material surface etching[13], rapid growth of silicon films, medical device sterilization[14], blood coagulation[15], cancer cell apoptosis[16], tissue regeneration, and wastewater treatment $[17,18]$.

The jet can be generated by different plasma sources, such as pulsed DC, AC, RF, and microwave. For ACdriven plasma jet, dielectric barrier discharge reactor can be operated at a wide range of applied voltage in atmospheric pressure.

\footnotetext{
* Corresponding author: 183375865@qq.com
} 
In the present work, a double-dielectric barrier discharge reactor is made for plasma jet generation. Charteristics of reactor, discharge power, temperature thermogram of plasma jet, and factors of plasma jet generation are experimentally investigated, and the sterilization efficiency of water and surface is evaluated.

\section{Experimental section}

Fig. 1 shows the experimental setup used in the present work, in which the system can be regarded as the Ar gas sources, plasma jet reactor, and detection part with several monitor instruments. The plasma jet is generated by a double-dielectric barrier discharge reactor with two cylindrical quartz tube of around $3 \mathrm{~mm}$ inner diameter and $1 \mathrm{~mm}$ inner diameter, respectively. The outward quartz tube is wrapped by a grounded electrode made of copper foil, and a copper rod high-voltage electrode of around $0.8 \mathrm{~mm}$ diameter is placed in the inner quartz tube. A high frequency AC high-voltage power source is used for energizing the reactor. The experiments are carried out at atmospheric pressure and ambient temperature. For plasma jet generation, the gas flow rate is $1 \mathrm{~L} / \mathrm{min}$ to $4 \mathrm{~L} / \mathrm{min}$ as the experiment needed.

The applied voltage is measured by a digital oscilloscope (DS1102E, Rigol, China) with a voltage probe (P5104, Tektronix, USA). The temperature distribution of the plasma jet reactor is measured by using an infrared camera (A35, FLIR, USA). The photographs of plasma jet are taken by the digital camera (EOS 7D, Canon, Japan). Discharge power $\mathrm{P}$ is measured by V-Q Lissajous method. The measurement circuit has been detailed described in our previous works[19].

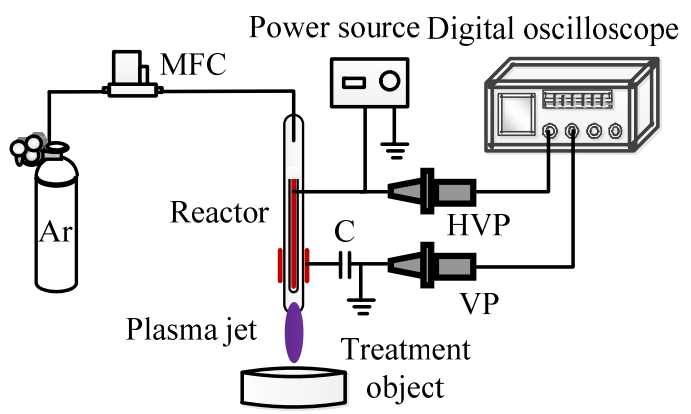

Fig. 1. Schematic diagram of experimental setup.

E. coli ATCC25922 is used for experiments. Their number density is obtained by means of heterotrophic plate count method. The E. coli ATCC25922 is used to evaluate the sterilization efficiency. It is firstly cultivated for $8-10 \mathrm{~h}$ at $310 \mathrm{~K}$ in a prepared Luria-Bertani (LB) medium which consists of $5 \mathrm{~g} / \mathrm{L}$ of yeast extract, $10 \mathrm{~g} / \mathrm{L}$ of peptone, and $10 \mathrm{~g} / \mathrm{L}$ of sodium chloride. Its number density rises to about $10^{7}-10^{9} \mathrm{cfu} / \mathrm{mL}$. Then, the E. coli is harvested by centrifugation (10000 rpm, $2 \mathrm{~min})$ and suspended in deionized water.

The sterilization efficiency in water is quantitatively investigated by treatment of ELISA plate with precoated bacteria liquid in the substrate. The ELISA plate has 48 holes with diameter of $6 \mathrm{~mm}$, depth of $11 \mathrm{~mm}$. For each hole, $250 \mu \mathrm{L} \mathrm{E}$. coli bacteria liquid is added. The distance between reactor and ELISA plate is $8 \mathrm{~mm}$. The surface sterilization capacity of plasma jet is analyzed qualitatively according to the plaque size of $E$. coli on solid ager plate. The diameter of the ager plate is $90 \mathrm{~mm}$, the spacing between the reactor and the ager plate is about $15 \mathrm{~mm}$.

Fig. 2 shows the typical growth curve of E. coli. The $\mathrm{OD}_{600}$ of the bacterial solution is about $1.6 \mathrm{~A}$ at the cultivated time of $8 \mathrm{~h}$. As a result, the density of the bacterial liquid is about $10^{7}-10^{9} \mathrm{cfu} / \mathrm{mL}$, indicating that the cell growth reached the logarithmic phase and the activity of the cells are in the strongest state. As showed in Fig. 3, the E. coli can be observed in the LB medium.

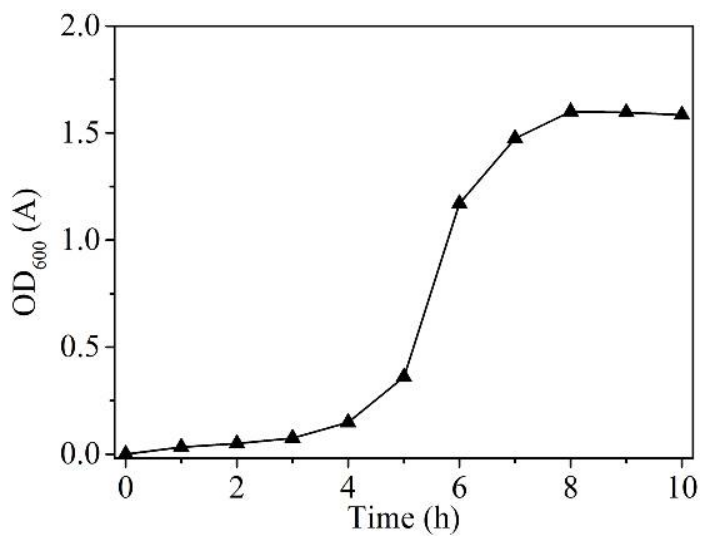

Fig. 2. Typical growth curve of E. coli.

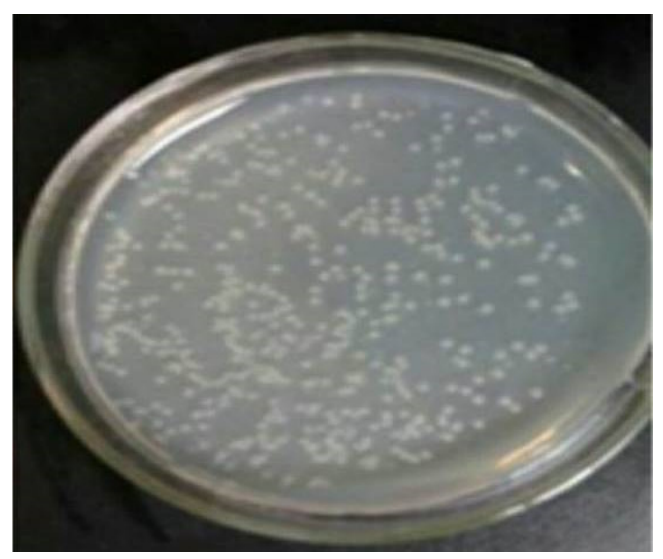

Fig. 3. Typical image of E. coli in the LB medium.

\section{Results and discussion}

\subsection{Plasma jet generation}

\subsubsection{Charteristics of reactor}

Fig. 4 shows the typical output voltage waveform of the AC power sources with frequency of $18.4 \mathrm{kHz}$ at the $\mathrm{Ar}$ flow rate of $1 \mathrm{~L} / \mathrm{min}$. It is obvious that the output of the high frequency AC power sources is stable.

Fig. 5 shows the relationship between applied voltage (Vp-p) and discharge power of the reactor with the 
voltage frequency of $18.4 \mathrm{kHz}$ and the Ar flow rate of 1 $\mathrm{L} / \mathrm{min}$. The discharge power increases gradually as the applied voltage increased. For the applied voltage of 3.6 $\mathrm{kV}, 5.6 \mathrm{kV}$ and $7.6 \mathrm{kV}$, the discharge power of the reactor is about $0.07 \mathrm{~W}, 0.17 \mathrm{~W}$, and $0.31 \mathrm{~W}$, respectively. It is obvious that the power of the plasma jet is very low.

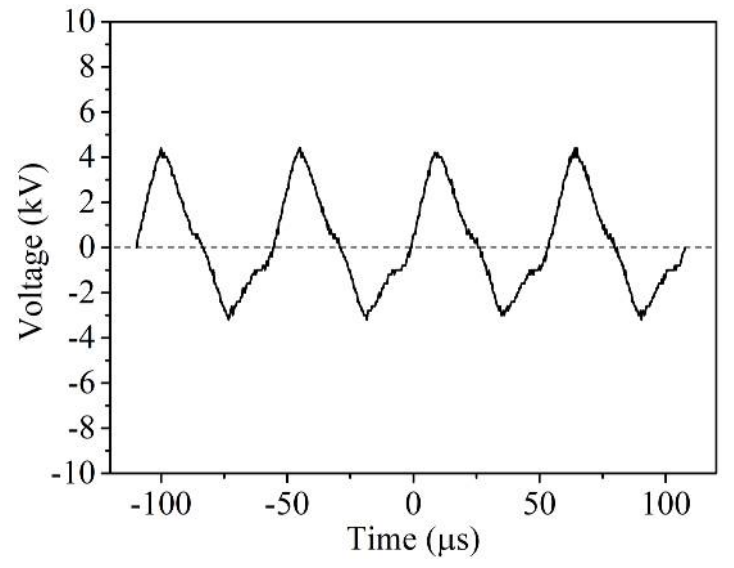

Fig. 4. Typical output voltage waveform of the AC power sources with frequency of $18.4 \mathrm{kHz}$.

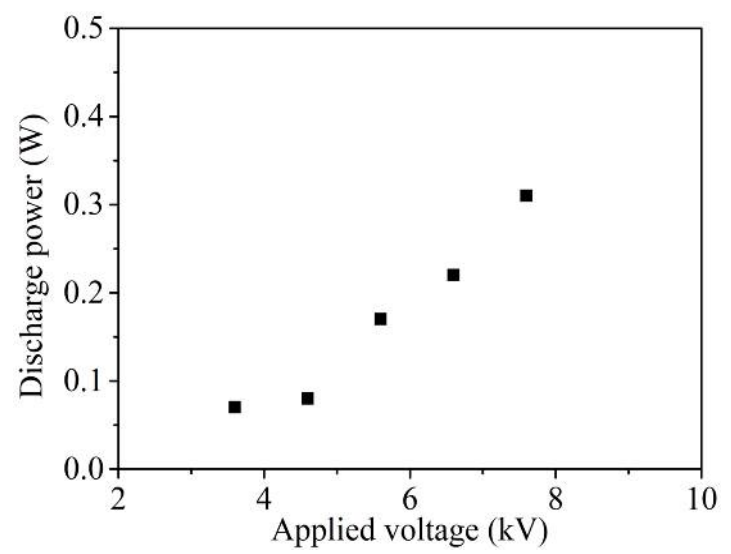

Fig. 5. The relationship between applied voltage $\left(V_{p-p}\right)$ and discharge power of the reactor.

For tissue or skin sterilization, non-thermal plasma is a smart solutions because of its chemical agents free process. Actually, plasma jet is a kind of non-thermal plasma with low gas temperature and energy density. As shown in Fig. 6, the temperature thermogram of the Ar plasma jet is measured using an infrared imager. It is obvious that the temperature of the jet is not high which is agree well with the low discharge power of the plasma reactor, for example, for the applied voltage of $4.6 \mathrm{kV}$, $5.6 \mathrm{kV}, 6.6 \mathrm{kV}$, and $7.6 \mathrm{kV}$, the max temperature of the jet is about $35.8{ }^{\circ} \mathrm{C}, 37.7{ }^{\circ} \mathrm{C}, 40.6{ }^{\circ} \mathrm{C}$, and $42.4{ }^{\circ} \mathrm{C}$, respectively. Meanwhile, the average temperature of the jet almost does not change with different applied voltage. This kinds of plasma jet can be used in the way of directly contact without thermal injury.

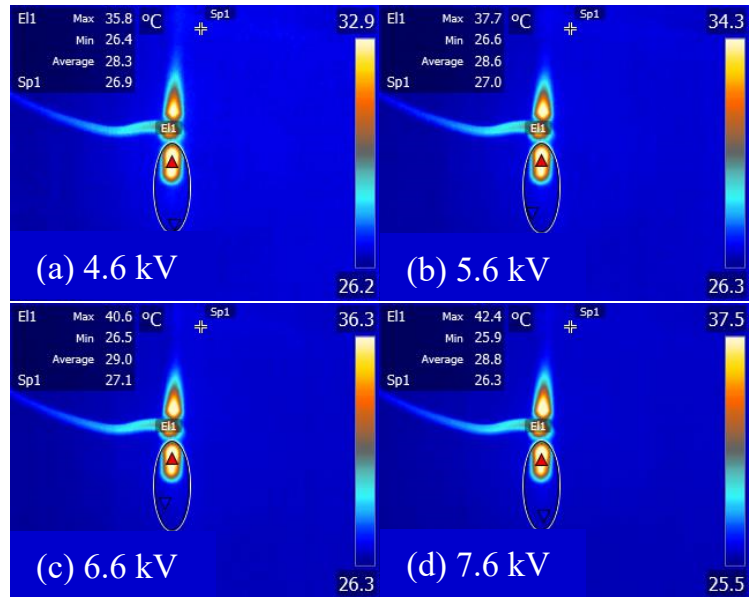

Fig. 6. The temperature thermogram of plasma jet with different applied voltage.

\subsubsection{Ar plasma jet}

Fig. 7 shows the effects of applied voltage on the plasma jet generation with the frequency of $18.4 \mathrm{kHz}$ and the $\mathrm{Ar}$ flow rate of $1 \mathrm{~L} / \mathrm{min}$. The length of plasma jet increases as the applied voltage increased. At the applied voltage of $4.6 \mathrm{kV}, 5.6 \mathrm{kV}, 6.6 \mathrm{kV}$ and $7.6 \mathrm{kV}$, the length of plasma jet is about $5 \mathrm{~mm}, 12 \mathrm{~mm}, 14 \mathrm{~mm}$, and $16 \mathrm{~mm}$, respectively. For the higher applied voltage, more Ar gas can be ionized.

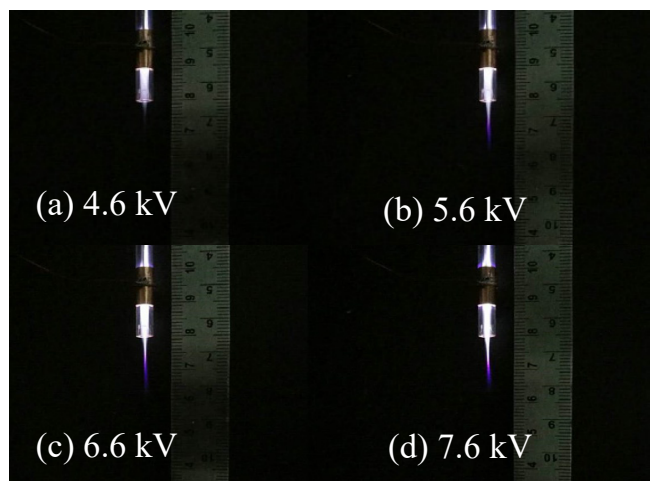

Fig. 7. Effects of applied voltage on the plasma jet generation.

Fig. 8 shows the effects of frequency on the plasma jet generation with the applied voltage of $7.6 \mathrm{kV}$ and the Ar flow rate of $1 \mathrm{~L} / \mathrm{min}$. The length of plasma jet increases as the frequency increased firstly, but when the frequency is as high as $18.4 \mathrm{kHz}$, the length of jet decreased. For example, at the frequency of $13.3 \mathrm{kHz}$, $15.2 \mathrm{kHz}$, and $18.4 \mathrm{kHz}$, the length of plasma jet is about $7 \mathrm{~mm}, 19 \mathrm{~mm}$, and $16 \mathrm{~mm}$, respectively.

Fig. 9 shows the effects of Ar flow rate on the plasma jet generation with the applied voltage of $7.6 \mathrm{kV}$ and the frequency of $18.4 \mathrm{kHz}$. It is obvious that the length of plasma jet increases as the gas flow increased firstly, and the longest of jet was observed at the gas flow of 3.5 $\mathrm{L} / \mathrm{min}$ which is about $19 \mathrm{~mm}$. But when the gas flow is as high as $4 \mathrm{~L} / \mathrm{min}$, the length of jet decreased because of the specific input energy become low that is not enough to maintain the discharge well. 


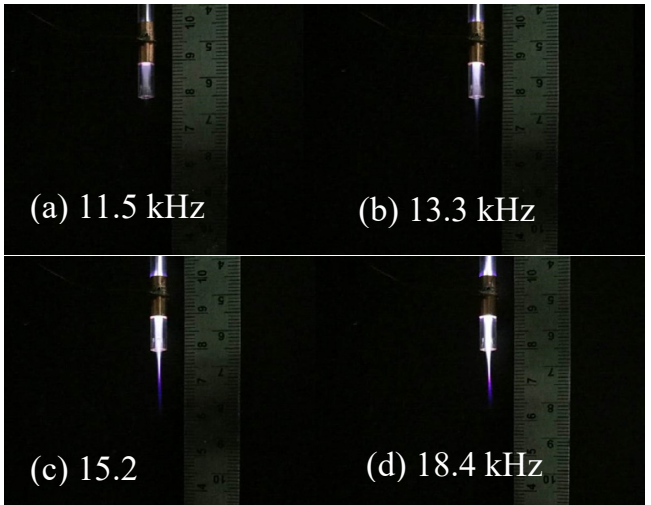

Fig. 8. Effects of frequency on the plasma jet generation.

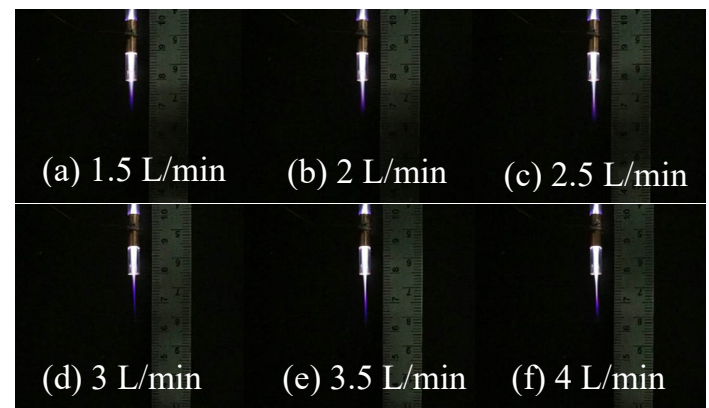

Fig. 9. Effects of gas flow on the plasma jet generation.

\subsection{Sterilization}

\subsubsection{Water sterilization}

Plasma jet has lots of active species that can be used for sterilization. Fig. 10 shows the effects of applied voltage on the water sterilization with the frequency of $18.4 \mathrm{kHz}$, the Ar flow rate of $2 \mathrm{~L} / \mathrm{min}$ and operation time of $3 \mathrm{~min}$. The contral sample with bacterial liquid in the ELISA plate is treated by the Ar flow without diacharge. There is no sterilization effect observed for Ar alone to treat the water with $E$. coli. The sterilization efficiency increases as the applied voltage increased. At the applied voltage of $7.6 \mathrm{kV}$, the sterilization efficiency is about $77.4 \%$.

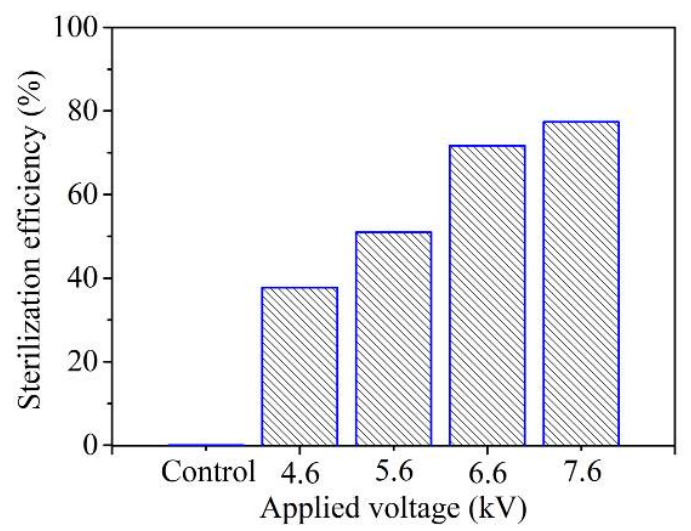

Fig. 10. Effects of applied voltage on the water sterilization.

Fig. 11 shows the effects of treatment duration on the water sterilization with the frequency of $18.4 \mathrm{kHz}$, the applied voltage of $5.6 \mathrm{kV}$, and the $\mathrm{Ar}$ flow rate of 2
$\mathrm{L} / \mathrm{min}$. The sterilization efficiency increases as the treatment time increased. There is about $73.6 \%$ of $E$. coli being sterilized after 5 min of plasma jet treatment. For a longer treatment duration, there is more active species come into the water which leads to a better sterilization effect.

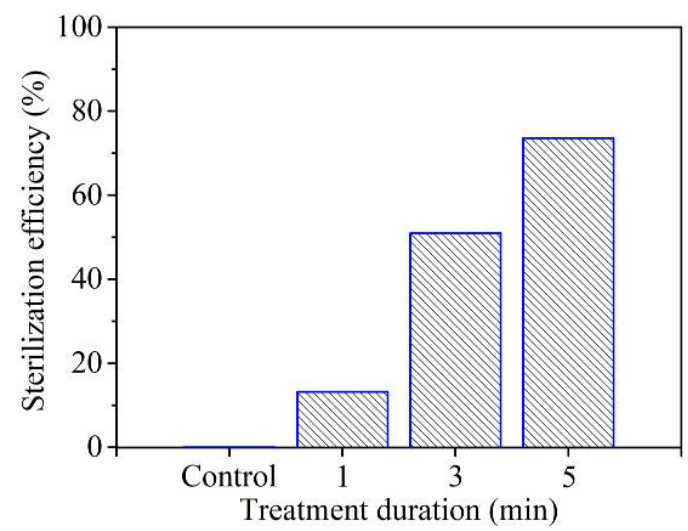

Fig. 11. Effects of treatment duration on the water sterilization.

\subsubsection{Surface sterilization}

Fig. 12 shows the effects of applied voltage on the surface sterilization with the frequency of $18.4 \mathrm{kHz}$, the Ar flow rate of $2 \mathrm{~L} / \mathrm{min}$ and operation time of $3 \mathrm{~min}$. The contral sample was treated by the $\mathrm{Ar}$ flow without diacharge, and it is obvious that the Ar alone has no sterilization effect. The the diameter of the plaque is gradually increased as the applied voltage increased.

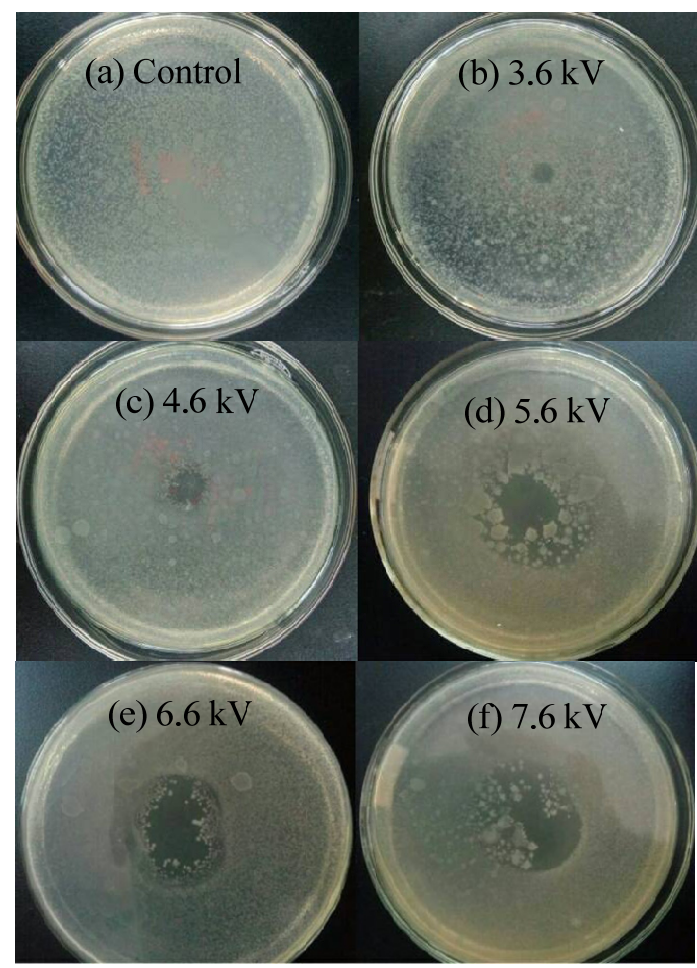

Fig. 12. Effects of applied voltage on the surface sterilization.

Fig. 13 shows the effects of treatment duration on the surface sterilization with the frequency of $18.4 \mathrm{kHz}$, the applied voltage of $5.6 \mathrm{kV}$, and the Ar flow rate of 2 
$\mathrm{L} / \mathrm{min}$. The the size of the plaque is gradually increased as the treatment time increased. The diameter of plaque is about $30 \mathrm{~mm}$ after $5 \mathrm{~min}$ of plasma jet treatment.

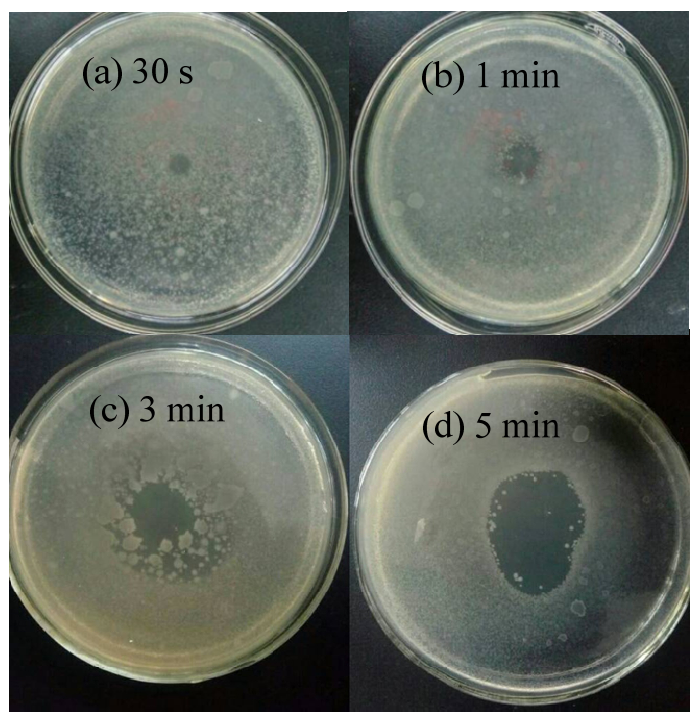

Fig. 13. Effects of treatment duration on the surface sterilization.

\subsection{Sterilization mechanism}

In the plasma process, there are lots of active species that can be used for sterilization. The charged particles and ROS, including $\mathrm{O}^{2-}, \mathrm{O}, \mathrm{O}_{3}, \mathrm{OH}, \mathrm{H}_{2} \mathrm{O}_{2}$, and metastable state $\mathrm{O}_{2}{ }^{*}$, are expected to play an important role in the sterilization of bacteria[20]. The excited molecules have no significant direct effect on the inactivation of bacteria. In the plasma jet, the intensity of UV light is low. Besides, the heat produced is little because of the low discharge power, so that the sterilization effect is negligible.

\section{Conclusions}

Characteristics of Ar plasma jet generation in an atmospheric pressure plasma reactor with doubledielectric barrier discharge are experimental investigated. The Ar plasma jet is generated stable in the reactor with low applied voltage and small discharge power. The length of Ar plasma jet increases as the applied voltage, frequency or gas flow increased, but plasma jet generation can be restricted in high frequency or gas flow. For the applied voltage of $7.6 \mathrm{kV}$ and the frequency of $18.4 \mathrm{kHz}$, the length of the plasma jet is about $19 \mathrm{~mm}$ at the gas flow of $3.5 \mathrm{~L} / \mathrm{min}$. For the conditions of frequency of $18.4 \mathrm{kHz}$, applied voltage of $5.6 \mathrm{kV}$, and flow rate of $2 \mathrm{~L} / \mathrm{min}$, the diameter of plaque is about $30 \mathrm{~mm}$ after $5 \mathrm{~min}$ of plasma jet treatment. With regard to water and surface sterilization, a higher applied voltage or longer treatment duration is preferred to improve its sterilization efficiency in this experiment.

\section{Acknowledgment}

This work is supported financially by the Natural Science Foundation of China (No. 21507043), Guangdong Natural Science Foundation (No. 2016A030307009, 2014A030310196, 2018A0303130089), Special Funds for the Cultivation of Guangdong College Students' Scientific and Technological Innovation ("Climbing Program" Special Funds) (No. pdjh2018b0471), and Guangdong Undergraduate Innovation and Entrepreneurship Training Program (No. 201810582032S). And supported by Foundation for Distinguished Young Talents in Higher Education of Guangdong, China (No. 2017GKQNCX096) and Yangjiang Polytechnic Applied Technology Collaborative Innovation Center Project.

\section{References}

1. E. Grawinski, Med. Weter. 51, 383 (1995)

2. X. Xue, R. Cheng, L. Shi, M. Kang, Y. Zhu, Science \& Technology Review. 34, 19 (2016)

3. L. H. Liegel, Turrialba. 36, 11(1986)

4. R. Lall, A. Sahu, A. Jaiswal, S. Kite, A. R. Sowmya and M. C. Sainath, J. Contemp. Dent. Pract. 19, 698 (2018)

5. R. R. ERNST, J. E. DOYLE, Biotechnol. Bioeng. 10, 1(1968)

6. J. Van Durme, J. Dewulf, C. Leys, H. Van Langenhove, Appl. Catal. B-Environ. 78, 324 (2008)

7. L. Nie, Y. Yang, J. Duan, F. Sun, X. Lu, G. He, J. Phys. D-Appl. Phys. 51, e34520434 (2018)

8. D. Y. Kim, S. J. Kim, H. M. Joh, T. H. Chung, Phys Plasmas. 25, e0735057 (2018)

9. E. Simoncelli, D. Barbieri, R. Laurita, A. Liguori, A. Stancampiano, L. Viola, Clinical Plasma Medicine. 3, 77 (2015)

10. G. Isbary, G. Morfill, H. U. Schmidt, M. Georgi, K. Ramrath, J. Heinlin, Brit. J. Dermatol. 163, 78 (2010)

11. T. von Woedtke, A. Kramer, K. Weltmann, plasma Process Polym. 5, 534 (2008)

12. O. Handorf, T. Weihe, S. Bekeschus, A. C. Graf, U. Schnabel, K. Riedel, Appl. Environ. Microbiol. 84, e01163-1821 (2018)

13. A. J. Knoll, P. Luan, A. Pranda, R. L. Bruce, G. S. Oehrlein, Plasma Process Polym. 15, e17002175 (2018)

14. C. Zheng, Y. Kou , Z. Liu , H. Jiang, Y. Huang, K. Yan, J. Bio. Eng. 34, 303 (2017)

15. K. Miyamoto, S. Ikehara, H. Takei, Y. Akimoto, H. Sakakita, K. Ishikawa, Arch. Biochem. Biophys. 605, 95 (2016)

16. S. J. Kim, T. H. Chung, Appl. Phys. Lett. 107, e0637026 (2015)

17. G. Deng, Q. Jin, S. Yin, C. Zheng, Z. Liu, K. Yan, Plasma Sources Sci. T. 20, 11550311 (2018) 
18. C. Zheng, Y. Xu, Y. Huang, Z. Liu, K. Yan, J. Zhejiang Univ. Eng. Sci. 48, 1329 (2014)

19. F. Feng, Y. Zheng, X. Shen, Q. Zheng, S. Dai, X. Zhang, Environ. Sci. Technol. 49, 6831 (2015)

20. X. Lu, T. Ye, Y. Cao, Z. Sun, Q. Xiong, Z. Tang, Z. Xiong, J. Hu, Z. Jiang, Y. Pan, J. Appl. Phys.104, 053309 (2008) 